\title{
Incidence of radius shortening following intramedullary nail fixation for gunshot fractures: a retrospective radiological audit
}

\author{
Michael Abramson, ${ }^{1,2 *}$ (D) Sithombo Maqungo, ${ }^{1,2}$ Roopam Dey, ${ }^{1,2}$ Maritz Laubscher ${ }^{1,2}$ \\ ${ }^{1}$ Department of Orthopaedic Surgery, Groote Schuur Hospital, University of Cape Town, Cape Town, South Africa \\ ${ }^{2}$ Orthopaedic Research Unit (ORU), Division of Orthopaedic Surgery, University of Cape Town, Cape Town, South Africa \\ ${ }^{*}$ Corresponding author: michaeljames1984@gmail.com
}

Citation: Abramson M, Maqungo S, Dey R, Laubscher $M$. Incidence of radius shortening following intramedullary nail fixation for gunshot fractures: a retrospective radiological audit. SA Ortho J 2021;20(4):226-230. http://dx.doi.org/10.17159/23098309/2021/v20n4a6

Editor: Prof. Nando Ferreira, Stellenbosch University, Cape Town, South Africa

Received: September 2020

Accepted: December 2021

Published: November 2021

Copyright: @ 2021 Abramson M. This is an open-access article distributed under the terms of the Creative Commons Attribution Licence, which permits unrestricted use, distribution and reproduction in any medium, provided the original author and source are credited.

Funding: No funding was received for this study.

Conflict of interest: The authors declare they have no conflicts of interest that are directly or indirectly related to the research.

\begin{abstract}
\section{Background}

Intramedullary nail fixation is an option to manage highly comminuted fractures of the radius shaft resulting from gunshot wounds. However, complications including nail migration and malunion are well documented. We have noticed some patients presenting to our clinic with radiological shortening following nail fixation. This may result in chronic pain, reduced pronation and supination, poor grip strength and early onset arthrosis. This study aimed to quantify the incidence of radiological shortening following fixation of isolated gunshot wound (GSW) fractures of the radius with an intramedullary nail. Our secondary objectives were to identify if length of the zone of comminution and anatomical location of the fractures were risk factors for shortening, and to assess whether shortening was a result of surgical error, or whether shortening occurred over time.
\end{abstract}

\section{Methods}

We performed a retrospective review of all $(n=40)$ isolated radius nails performed between January 2012 and January 2019. Two doctors assessed the latest anterior-posterior forearm X-ray of every patient, using the rule of perpendiculars to calculate ulnar variance (UV). Shortening was defined as a UV $>5.0 \mathrm{~mm}$. If the radius was deemed shortened by consensus, then the immediate postoperative X-ray was also assessed to gauge when shortening occurred. Anatomical location in thirds and length of comminution $(\mathrm{mm})$ were also assessed.

\section{Results}

Forty patients with a mean age of 32 years (range 15-59) were included. Twelve patients' radiuses were assessed as radiologically short. All 12 were deemed to have been fixed short. One case shortened further over time. We found the incidence of shortening being dependent on the fracture location $(p=0.03)$, with the fractures occurring in the middle third of the arm shortening more. The measured zone of comminution between the shortened and non-shortened groups was not found to be statistically significant $(p=0.55)$.

\section{Conclusion}

The radius nail remains useful to manage comminuted radius shaft fractures following GSW. Meticulous technique is needed to avoid radiological shortening, seen in $30 \%$ of our series. This can lead to chronic pain, reduced grip strength and early onset arthrosis. We found no evidence that shortening develops over time. We found that the incidence of shortening is dependent on fracture position but did not find any causative relationship between length of the zone of fracture comminution and shortening.

Level of evidence: Level 4

Keywords: radial nail, shortening, gunshot

\section{Introduction}

Fractures of the forearm resulting from gunshot wounds (GSW) are often highly comminuted, involving a sizable bone segment, and can be very challenging to treat. ${ }^{1}$ The new generation locking intramedullary (IM) nail is an option to manage these highly comminuted fractures. ${ }^{2,3}$ Minimal disruption to the soft tissue envelope together with its stress-shielding biomechanical properties makes the IM nail an attractive alternative to conventional bridge plate techniques. ${ }^{4,5}$ The IM nail is not without its problems, and issues such as nail migration and malunion have been highlighted in the literature..$^{6,7}$

At our institution we have been using interlocking IM nails for isolated gunshot fractures of the radius since 2012. At follow-up we have noted some patients presenting with radius shortening following nail fixation to treat GSW fractures. Radius shortening is associated with clinical sequelae such as chronic pain, reduced 
pro- and supination, poor grip strength and early onset arthrosis of the distal radioulnar and radiocarpaljoints. . $^{811}$

Our study aimed to quantify the incidence of radiological radius shortening following radius nails for GSW fractures at our institution. Our secondary objectives were to identify whether the radius was fixated in a shortened position or whether it shortened over time. We also tried to identify any risk factors for radius shortening, specifically looking at whether length of the zone of comminution or anatomical location of the fractures contribute to its incidence.

\section{Patients and methods}

We performed a retrospective review of all $(n=40)$ isolated radius nails for GSW fractures performed at our institution between January 2012 and January 2019. We used the database from the nail provider (Afmed Pty Ltd) to confirm all cases were included in the study. Any radius nail with an ipsilateral fracture of the ulna that required fixation was excluded.

The latest AP forearm X-ray of each patient was assessed by two surgeons experienced in orthopaedic trauma. The rule of perpendiculars was used to calculate ulnar variance (UV) ${ }^{12}$ (Figure 1). All X-rays were assessed on our Philips Intellispace digital PACS (Philips, Netherlands). Shortening was defined in this study as a positive UV greater than $5.0 \mathrm{~mm}$. If the radius was deemed to be short by consensus using this criterion, then the immediate postoperative X-ray was also assessed to gauge if the fracture was fixed short or if shortening developed over time. This $X$-ray was taken within 48 hours of surgery. All the cases where index X-rays were deemed not to be shortened were followed up radiologically to confirm that they remained outside the criteria for shortening stipulated in the study. No patients were found to cross over from the normal into the shortened category over the study period. Anatomical location (dividing radius shaft into thirds) and length of the zone of comminution in millimetres were assessed by two independent assessors (and a third in cases of disagreement).

The measurements were segregated into short and not-short groups. The distribution of the data was checked using the ShapiroWilk test. Normally distributed data were compared using student's t-test, and non-normally distributed data were compared using Mann-Whitney $U$ test. Pearson chi-squared test and Fisher's exact test were appropriately used to find the dependence of shortening with the degree of fracture comminution and fracture location. The condition for statistical significance was set at $p<0.05$. All the statistical analyses were performed using IBM SPSS v.26 (IBM Corp, Armonk, NY, USA).

\section{Surgical technique}

The procedure is performed with the patient supine on a radiolucent arm table, with the aid of a tourniquet. Prior to commencing the operation, the contralateral forearm is screened to pre-select the appropriate length and diameter of the nail, as well as to assess the patient's baseline UV in order to try replicate a similar UV on the affected side. There are three different length nails available (Acumed, USA), ranging from 190 to $230 \mathrm{~mm}$. When deciding between two different lengths, we used the shorter of the two to avoid the possibility of a proud nail distally.

The entry point, just ulnar to Listers tubercle and $5 \mathrm{~mm}$ proximal to the articular surface, is found with the aid of fluoroscopy. A $1 \mathrm{~cm}$ dorsally based longitudinal incision is then made, and blunt dissection performed down to the bone, taking care to avoid injury to the extensor pollicus longus tendon or a small branch of the radial sensory nerve that may cross the surgical field. The near cortex is breached with the entry awl, paying careful attention to the AP and lateral direction of the awl. Inaccuracy of these initial steps, particularly in the AP plane, can result in a malreduction of the radius bow. Directing the awl in a slight radial direction can aid in avoiding

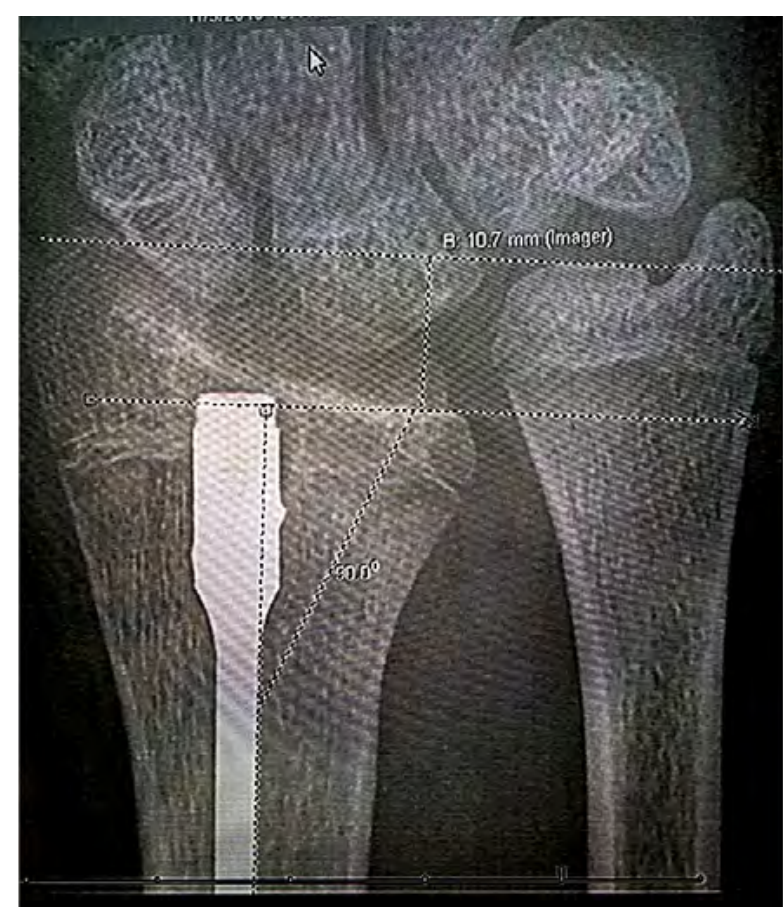

Figure 1. An example of how to measure ulnar variance: Draw a line tangential to the articular surface of the ulnar. Draw a second line tangential to lunate fossa of radius and perpendicular to shaft. Measure the distance between these two lines. In this example, the UV is measured at $10.7 \mathrm{~mm}$, and would be considered shortened in this study (normal: UV -4.1 to +2.3 ).

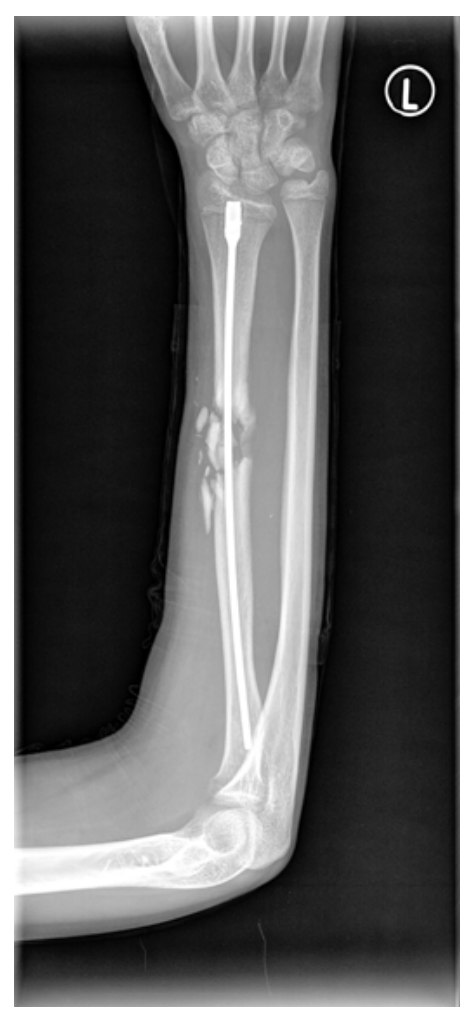

Figure 2. An AP X-ray of a patient, taken three months post surgery. Note the significant ulnar plus (measured at $11.7 \mathrm{~mm}$ ). She presented with functional loss of supination, requiring revision surgery.

Table I: The distribution of fracture occurrences and the incidences of shortening across the proximal, middle and distal region of the radius bone

\begin{tabular}{lcc} 
Region & Fracture (\%) & Shortening (\%) \\
Proximal & 48 & 17 \\
\hline Middle & 40 & 58 \\
\hline Distal & 13 & 25 \\
\hline
\end{tabular}




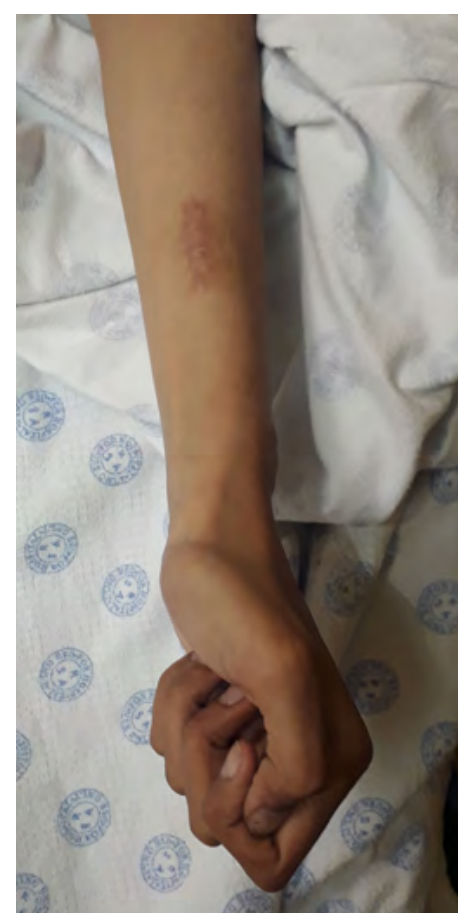

Figure 3. Same patient as in Figure 2 , demonstrating her maximum active supination of the affected side

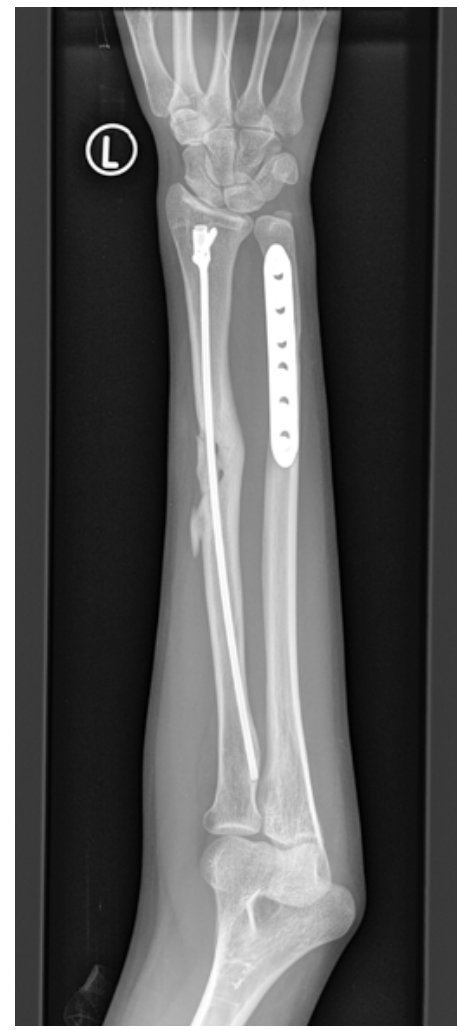

Figure 5. An AP X-ray of the same patient (Figure 2), following an ulnar shortening osteotomy and plating aimed at restoring her native distal radial ulnar joint relationship

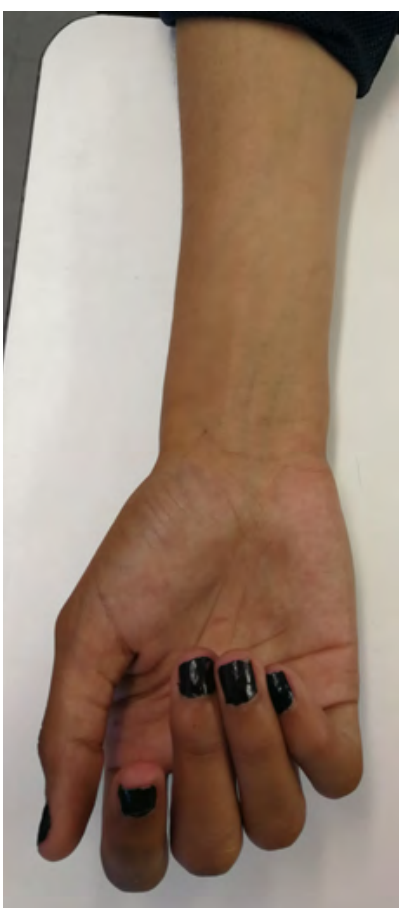

Figure 4. Same patient as in Figure 2, demonstrating her maximum active supination of the normal side

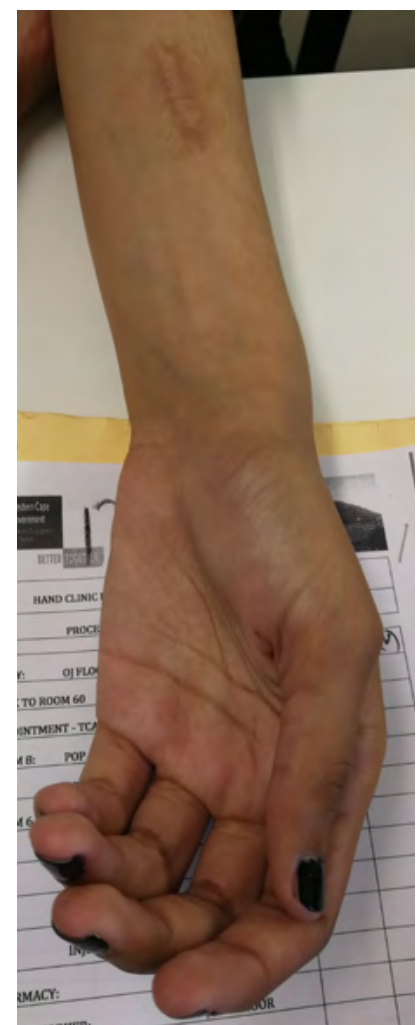

Figure 6. Photo demonstrating patient's (Figure 2) improved supination range of motion, six weeks post ulnar shortening procedure

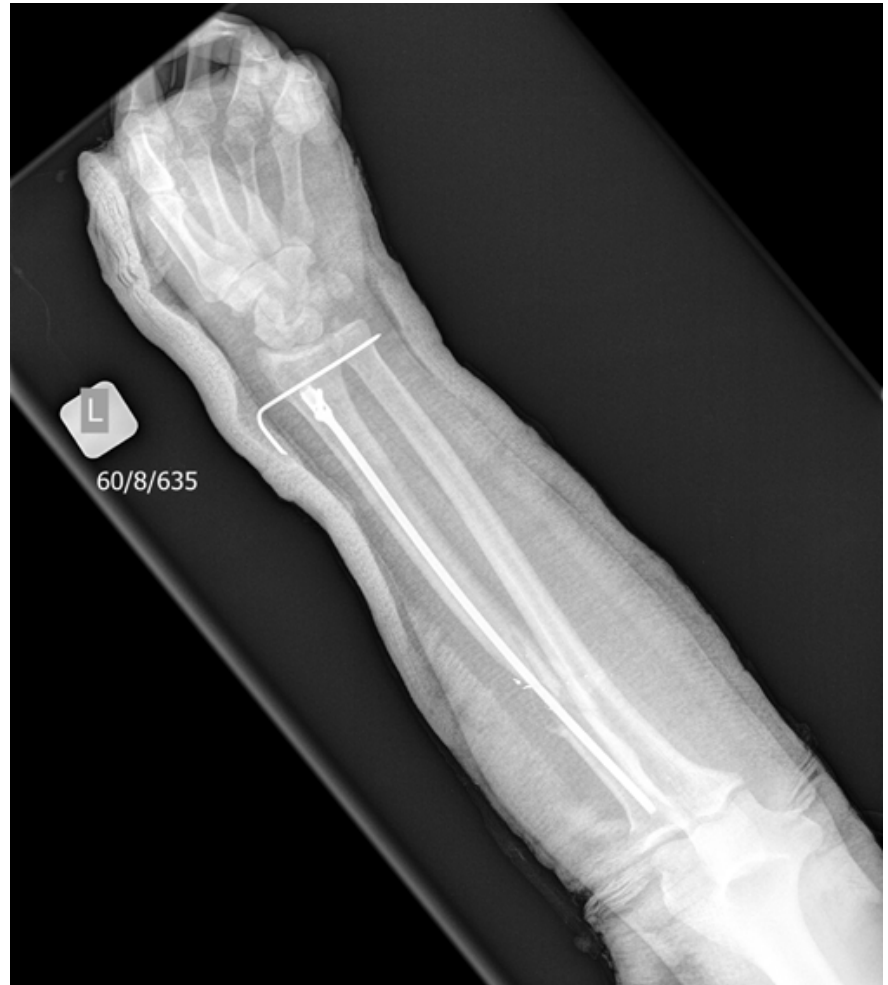

Figure 7. AP X-ray of a case where a K-wire was used to maintain the length of the distal radioulnar joint

this error. The $3.1 \mathrm{~mm}$ reamer is then inserted into the medullary canal, the fracture reduced, and the reamer passed across the fracture, stopping at level of the biceps tuberosity. Depending on the ease of the pass, and the preoperative radiological assessment of the bone's canal diameter, the surgeon may choose to pass the $3.7 \mathrm{~mm}$ reamer to enlarge the medullary cavity to aid in the insertion of a larger calibre nail. The appropriately selected nail is then passed, making sure the fluted nail tip is seated firmly in the subchondral bone proximally to improve purchase. Tapping of the nail using a mallet is often needed to ensure adequate seating of the nail. Restoration of radius length is then achieved radiologically, and rotation confirmed using Evans rule, which states that the biceps tuberosity and radial styloid should be visualised $180^{\circ}$ to one another in a normally rotated radius. ${ }^{13}$ The nail is then locked with the forearm in supination, using a single dorsal $3.5 \mathrm{~mm}$ locking screw. Blunt dissection down to the bone is needed to avoid injury to important structures including the extensor tendons or a branch of the superficial radial sensory nerve. Careful intraoperative screening is needed post insertion to ensure the screw is not proud, as this may lead to extensor and flexor tendon irritation. An examination under fluoroscopy is performed to confirm whether the fixation is rotationally stable. If not, an above-elbow slab is used to limit pro- and supination, for a minimum of four weeks, or until soft callus is seen on X-ray.

\section{Results}

Forty patients met the criteria for inclusion in the study. No cases were excluded from analysis. Thirty-seven (93\%) were male, with a mean age of 32.6 years (range $15-59)$. Twelve cases (30\%) were assessed as radiologically short by both assessors. The mean shortening of the affected group was $7.4 \mathrm{~mm}$ (range 5.1-13.0). All 12 were deemed to have been fixed in a shortened position. One case was assessed to further shorten over time by approximately $2 \mathrm{~mm}$. 
Most fractures affected the proximal and middle third of the radius. The anatomical distribution of all the fractures was five $(13 \%)$ distal, $16(40 \%)$ middle and $19(48 \%)$ proximal. The majority $(58 \%)$ of all shortened fractures were found in the middle third of the radius. Postoperative shortening had a statistically significant $(p=0.03)$ dependency on the position of fracture. Refer to Table I for a summary of results.

We found no statistically significant $(p=0.55)$ difference between the average length of the zone of comminution $(41.6 \pm 25.9 \mathrm{~mm}$ in the shortened group and $35.6 \pm 18.9 \mathrm{~mm}$ in the not-shortened group) and the incidence of shortening between the two groups.

\section{Discussion}

The surgical goals of any forearm shaft fracture fixation are the restoration of axial and rotational alignment, maintaining stability of the proximal and distal radioulnar joints and early range of motion. 1,3 $^{1,}$ For most forearm fractures, compression plating remains the gold standard, as anatomical reduction and primary bone healing are considered fundamental in achieving the best functional outcome. ${ }^{3}$ Complications associated with plate fixation include a $0.8-2.3 \%$ risk of infection, ${ }^{14}$ attributed to the extensive soft tissue dissection required, and up to $30 \%$ refracture rate when the plate is removed. ${ }^{15}$

Fracture comminution however, a constant feature following GSW, ${ }^{1}$ poses unique treatment challenges and precludes primary bone healing strategies in favour of secondary bone healing. Secondary bone healing can be achieved either by nonoperative means with the use of a plaster cast, or surgically, utilising either bridge plating techniques or IM nailing. Nonoperative management of adult diaphyseal radius fractures is associated with high complication rates, most notably failure to maintain reduction, radius shortening and stiffness. ${ }^{5}$

The benefits of an intramedullary device over bridge plating include a smaller incision, improved cosmesis and less soft tissue dissection, while also maintaining a more optimal environment for healing due to minimal disruption to the fracture haematoma. ${ }^{6,7}$ Its stress-sharing biomechanics also promote a predictably stronger fracture callus compared to bridge plating. ${ }^{6}$

The early radial nails were criticised for their potential to result in iatrogenic injury to the posterior interosseus nerve during proximal locking. ${ }^{6}$ This led to the development of the currently available radial nail, which has a flanged proximal end, eliminating the need for proximal locking while still providing rotational control through a press-fit mechanism into the unreamed subchondral bone. This requires attention to detail in the reaming step of the surgery (see surgical technique) as reaming beyond the bicipital tuberosity can potentially reduce the proximal purchase into the subchondral bone and therefore reduce the rotational control the nail provides. The modern nail is not without problems either, with nail migration and malunion (failure to restore radial bow) have been highlighted. ${ }^{14}$

Many authors have investigated the clinical and biomechanical consequences of radius shortening. Crisco et al. reported that radius shortening changes the axis of rotation of the forearm, resulting in reduced joint congruency, reduction in rotation and changes in stress across the triangular fibrocartilage complex (TFCC) ${ }^{16}$ Adams concluded that the greatest change in kinematics at the distal radius ulnar joint is due to radius shortening, which results in increased strain in the articular disc, and volar and dorsal radioulnar ligaments of the TFCC, leading to reduced grip strength. ${ }^{17}$ They also reported that radius shortening results in changes in radiocarpal and radioulnar joints and that radius shortening has the most direct correlation with increased symptoms, including an increased risk of arthrosis. Lidstrom concluded their work by claiming that restoring radius length is the most important factor associated with a good clinical outcome. ${ }^{18}$
Fernandez showed that radius shortening can result in reduced pro- and supination. ${ }^{19}$ One of the patients in our series presented for her follow-up with significantly reduced supination, affecting her activities of daily living. Her postoperative $\mathrm{X}$-rays show a positive ulnar variance of $11.7 \mathrm{~mm}$ (Figure 2). Comparative pictures shown in Figures 3 and 4 demonstrate her limitation in supination compared to the unaffected side. She underwent an ulnar shortening osteotomy (Figure 5) to alleviate some of her symptoms. Figure 6 shows her improved clinical picture, six weeks post revision surgery. Distal radioulnar joint pain, ulnar impaction syndrome, TFCC tears and early onset osteoarthritis are the other commonly associated conditions attributed to radius shortening. . $^{8,9,16}$

Ulnar variance (UV) is a commonly used radiological method to assess for radius shortening. Plain radiographs are proven to be acceptably accurate to measure UV. ${ }^{10,12}$ Being a dynamic measurement, it is influenced by several factors including rotation, grip and beam direction, ${ }^{20}$ and therefore, normal reference ranges differ. While international consensus on UV ranges is lacking, most quoted studies of UV in a neutral, relaxed position is $0.9 \mathrm{~mm}$ (range $-4.1 \mathrm{~mm}$ to $+2.3 \mathrm{~mm}$ ). ${ }^{10}$ To accommodate for magnification error, potential positional variability when taking the X-ray, and to allow for the potential of a patient having a pre-existing ulnar plus of up to $2.5 \mathrm{~mm}$, we defined radiological shortening as more than or equal to $5 \mathrm{~mm}$ positive UV. We used the rule of perpendiculars to measure UV, having been shown to have the best interobserver reliability of the three commonly practiced methods of assessing. ${ }^{10,12,21}$

There is ample published literature on the clinical impact of a positive UV. Most of the literature suggests that $2.5 \mathrm{~mm}$ is the amount of radius shortening beyond which clinical symptoms develop. ${ }^{8-10,22,23}$ Early onset osteoarthritis due to increase in the mechanical load though the ulnar, ${ }^{20}$ ulnar impaction syndrome, distal radius ulnar joint (DRUJ) pain/degeneration, reduced grip strength and carpal instability are also reported in the literature.8,9,17,22

We could only find two articles that specifically looked at IM nails in the setting of GSW. In the first, the author makes mention of a case where DRUJ shortening occurs over the course of the healing process but does not elaborate further on its clinical significance. ${ }^{7}$ The second paper is an outcome-based study and does not specifically look at radius shortening. ${ }^{1}$

While formulating this study, we hypothesised that highly comminuted radius shaft fractures would shorten over time. We felt the mechanical properties of the IM nail may not be adequate to maintain length for sufficient time to allow union, as biomechanical studies have proven them inferior to plates. ${ }^{5}$ The results of our study, however, seem to suggest that, should meticulous care be taken intraoperatively to restore the correct radius length before locking the nail, that shortening does not occur over time.

We therefore advocate the following steps to ensure shortening is avoided. First, take measurements of the contralateral radius for length and diameter in order to plan which available nail will give you the optimal proximal subchondral purchase, without leaving it proud distally. If only a single distal locking screw is available, inadequate purchase of the fluted blade tip into unreamed bone may render the nail length unstable. Pay careful attention to the relationship of the distal radius and ulnar of the unaffected side in order to reproduce it on the operated side. Intraoperatively, the authors feel that tapping in of the nail to get adequate subchondral purchase may inadvertently result in shortening. To mitigate this potential problem, one of our surgeons held the reduced DRUJ out to length before inserting the nail using a K-wire prior to the insertion of the nail (Figure 7). The wire was left in-situ for three weeks, and the patient healed in an acceptable position. Given what we now know about the nail's seemingly consistent ability to hold fractures out to length if acceptably reduced, we feel that removing the wire at the conclusion of the surgery, thus limiting 
the potential side effects of an in-situ wire, is appropriate. Lastly, we wish to stress the importance of screening for stability at the conclusion of the surgery, and if there is any doubt, liberal use of an above-elbow back slab for four weeks, or until soft callus seen on X-ray, is advocated.

The secondary outcome measures of the study aimed to determine if length of comminution or anatomical location of the fracture had any direct correlation with an increased incidence of shortening. While shortening was found to be dependent on the fracture position, with a predilection for the middle third of the radius, the length of the zone of fracture comminution was found to not be a significant contributing factor.

The study is enhanced by its relatively large sample size, given the sparsity of this surgery, but it is limited by its retrospective design. Being a retrospective review, we could not ensure all the $X$-rays were standardised, thus potentially affecting the dynamic nature of UV. As detailed in the discussion section, we attempted to overcome this by setting a relatively high positive UV as our cut-off to define shortening. Finally, we are aware of the limitations in our detection methods for identifying and defining radius shortening, due mainly to a lack of formal contralateral control X-rays showing the patients' baseline UV. Obtaining intraoperative radiographs upon completion of the surgeries would have removed the remote possibility that the fracture was fixed correctly and shortened in the immediate postoperative period. While screening the contralateral side is an important intraoperative step, its accuracy in UV measurements for the purposes of this study is insufficient.

\section{Conclusion}

The radius nail remains a useful tool to manage comminuted radius shaft fractures following GSW, yet careful attention to detail is needed intraoperatively to avoid radiological shortening, as seen in $30 \%$ of our case series. We found no evidence of subsequent shortening if the radius length is corrected before the nail is locked. We also did not find any causative relationship between length of the zone of fracture comminution and subsequent shortening but found a dependent relationship between fracture location and incidence of shortening.

\section{Ethics statement}

The authors declare that this submission is in accordance with the principles laid down by the Responsible Research Publication Position Statements as developed at the 2nd World Conference on Research Integrity in Singapore, 2010.

Full ethics approval from the University of Cape Research Committee (HREC 580/2019) was obtained for this study.

All procedures were in accordance with the ethical standards of the responsible committee on human experimentation (institutional and national) and with the Helsinki Declaration of 1975 , as revised in 2008.

\section{Declaration}

The authors declare authorship of this article and that they have followed sound scientific research practice. This research is original and does not transgress plagiarism policies.

\section{Author contributions}

MA: Study conceptualisation, study design, data capture, data analysis, first draft preparation, manuscript revision, manuscript preparation

SM: First draft preparation, manuscript revision

RD: Data capturing, statistical analysis

ML: Manuscript revision, study design, study conceptualisation, manuscript preparation

\section{ORCID}

Abramson M (C) https://orcid.org/0000-0001-6677-3506 Maqungo S https://orcid.org/0000-0002-8735-8341 Dey R (1) https://orcid.org/0000-0002-3616-1995 Laubscher M ifttps://orcid.org/0000-0002-5989-8383

\section{References}

1. Maqungo S, Kauta N, McCollum G, Roche S. Clinical outcome of isolated low velocity gunshot radius fractures treated with closed reduction and locked intramedullary nailing. Bone Jt Surg - Orthop Proc. 2018;96-B(Suppl 19).

2. Weckbach $A, B$ Battert TR, Weißer $C$. Interlocking nailing of forearm fractures. Arch Orthop Trauma Surg. 2006;126(5):309-15

3. Saka G, Saglam N, Kurtulmuş T, et al. New interlocking intramedullary radius and ulna nails for treating forearm diaphyseal fractures in adults: A retrospective study. Injury. 2014;45(Suppl 1):S16-S23.

4. Hong G, Cong-Feng L, Chang-Qing Z, et al. Internal fixation of diaphyseal fractures of the forearm by interlocking intramedullary nail: short-term results in eighteen patients. J Orthop Trauma. 2005;19(6):384-91.

5. Rehman S, Sokunbi G. Intramedullary fixation of forearm fractures. Hand Clin. 2010;26(3):391-401.

6. Ozkaya U, Kilic A, Ozdogan U, et al. Comparison between locked intramedullary nailing and plate osteosynthesis in the management of adult forearm fractures. Acta Orthop Traumatol Turc. 2009;43(1):14-20.

7. Gelbart B, Aden A. Evaluation of intramedullary nailing in low-velocity gunshot wounds of the radius and ulna. SA Orthop J. 2013;12(3):35-41.

8. Pogue DJ, Viegas SF, Patterson RM, et al. Effects of distal radius fracture malunion on wrist joint mechanics. J Hand Surg Am. 1990;15(5):721-27.

9. Bu J, Patterson RM, Morris R, et al. The effect of radial shortening on wrist joint mechanics in cadaver specimens with inherent differences in ulnar variance. J Hand Surg Am. 2006;31(10):1594-600

10. Laino DK, Petchprapa CN, Lee SK. Ulnar variance: Correlation of plain radiographs, computed tomography, and magnetic resonance imaging with anatomic dissection. J Hand Surg Am. 2012;37(1):90-97.

11. Miyake J, Murase T, Yamanaka Y, et al. Three-dimensional deformity analysis of malunited distal radius fractures and their influence on wrist and forearm motion. J Hand Surg Eur Vol. 2012;37(6):506-12

12. Steyers CM, Blair WF. Measuring ulnar variance: A comparison of techniques. J Hand Surg Am. 1989;14(4):607-12.

13. Evans EM. Rotational deformity in the treatment of fractures of both bones of the forearm. $J$ Bone Jt Surg. 1945;27(3):373-79.

14. Lee YH, Lee SK, Chung MS, et al. Interlocking contoured intramedullary nail fixation for selected diaphyseal fractures of the forearm in adults. J Bone Jt Surg. 2008:90(9):1891-98.

15. Nagy L, Jankauskas L, Dumont CE. Correction of forearm malunion guided by the preoperative complaint. Clin Orthop Relat Res. 2008;466(6):1419-28.

16. Crisco JJ, Moore DC, Marai GE, et al. Effects of distal radius malunion on distal radioulnar joint mechanics - An in vivo study. J Orthop Res. 2007;25(4):547-55.

17. Adams BD. Effects of radial deformity on distal radioulnar joint mechanics. J Hand Surg Am. 1993;18(3):492-98

18. Lidström A. Fractures of the distal end of the radius: a clinical and statistical study of end results. Acta Orthop Scand. 1959;30(Suppl 41):1-118.

19. Fernandez DL. Should anatomic reduction be pursued in distal radial fractures? J Hand Surg Br. 2000;25(6):523-27.

20. Tomaino MM. The importance of the pronated grip $\mathrm{x}$-ray view in evaluating ulnar variance. $\mathrm{J}$ Hand Surg Am. 2000;25(2):352-57.

21. Thuysbaert $G$, Ringburg A, Petronilia $S$, et al. Measurement of ulnar variance and radia inclination on $\mathrm{X}$-rays of healed distal radius fractures. With the axis of the distal radius or ulna? Acta Orthop Belg. 2015;81(2):308-14.

22. Palmer A, Werner F. Biomechanics of the distal radioulnar joint. Clin Orthop Relat Res. 1984;187:26-35.

23. Dumont CE, Pfirmann CWA, Ziegler D, Nagy L. Assessment of radial and ulnar torsion profiles with cross-sectional magnetic resonance imaging: A study of volunteers. J Bone Jt Surg Am. 2006;88(7):1582-88. 